\title{
DBA/2 Mouse
}

National Cancer Institute

\section{Source}

National Cancer Institute. DBA/2 Mouse. NCI Thesaurus. Code C14604.

Derived from crosses made by Little in 1929-1930 between DBA progenitors. The DBA/2 mouse has a d H2 haplotype and carries the Cdh23ahl mutation that results in a progressive hearing loss beginning at 3-4 weeks of age and severe hearing loss after 3 months of age. Alleles GpnmbR150X and Tyrp1 isa contribute to an eye phenotype that closely resembles human hereditary glaucoma. The DBA/2 mouse strain shows severe intolerance to alcohol and morphine and is naturally CD94 deficient. 